\title{
GENERATION OF DIAGONAL ACTS OF SOME SEMIGROUPS OF TRANSFORMATIONS AND RELATIONS
}

\author{
Peter Gallagher and Nik RušKuc
}

The diagonal right (respectively, left) act of a semigroup $S$ is the set $S \times S$ on which $S$ acts via $(x, y) s=(x s, y s)$ (respectively, $s(x, y)=(s x, s y))$; the same set with both actions is the diagonal bi-act. The diagonal right (respectively, left, bi-) act is said to be finitely generated if there is a finite set $A \subseteq S \times S$ such that $S \times S=A S^{1}$ (respectively, $S \times S=S^{1} A, S \times S=S^{1} A S^{1}$ ).

In this paper we consider the question of finite generation for diagonal acts of certain infinite semigroups of transformations and relations. We show that the semigroups of full transformations, partial transformations and binary relations on an infinite set each have cyclic diagonal right and left acts. The semigroup of full finiteto-one transformations on an infinite set has a cyclic diagonal right act but its diagonal left act is not finitely generated. The semigroup of partial injections on an infinite set has neither finitely generated diagonal right nor left act, but has a cyclic diagonal bi-act. The semigroup of bijections (symmetric group) on an infinite set does not have any finitely generated diagonal acts.

\section{INTRODUCTION}

If $S$ is a semigroup and $X$ is a set then we say that $S$ acts on $X$ ( $X$ is a right $S$-act) via $\alpha: X \times S \rightarrow X(\alpha:(x, s) \mapsto x s \in X)$ if $\left(x s_{1}\right) s_{2}=x\left(s_{1} s_{2}\right)$ for all $x \in X, s_{1}, s_{2} \in S$. Left acts are defined analogously. We say that $X$ is a bi-act if $S$ acts on it both on the left and on the right and $(s x) t=s(x t)$ for all $x \in X, s_{1}, s_{2} \in S$.

If $S$ is a right (respectively, left, bi) $S$-act then $A \subseteq X$ is a generating set for this act if $A S^{1}=X$ (respectively, $S^{1} A=X, S^{1} A S^{1}=X$ ). If $A$ can be chosen to be finite then $X$ is said to be finitely generated. If $A$ can be chosen to be a singleton then we say that $X$ is cyclic.

The diagonal right act of $S$ is the set $X=S \times S$ with $S$ acting via $(x, y) s=(x s, y s)$. The diagonal left act is defined analogously, via $s(x, y)=(s x, s y)$. The diagonal bi-act is the same set $S \times S$ on which $S$ acts from both left and right.

Received 5th April, 2005

The authors are grateful to an anonymous referee for his/her suggestions for streamlining the proof of Theorem 4.3.

Copyright Clearance Centre, Inc. Serial-fee code: 0004-9727/05 \$A2.00+0.00. 


\begin{tabular}{|l|c|c|c|}
\hline Semigroup & Right act & Left act & Bi-act \\
\hline \hline $\mathcal{B}_{X}$ & cyclic (Thm 2.1) & cyclic (Thm 3.1) & cyclic \\
\hline $\mathcal{P}_{X}$ & cyclic (Cor 2.2) & cyclic (Cor 3.3) & cyclic \\
\hline $\mathcal{T}_{X}$ & cyclic (Cor 2.3) & cyclic (Thm 3.2) & cyclic \\
\hline $\mathcal{F}_{X}$ & cyclic (Cor 2.4) & infinite (Thm 3.4) & cyclic \\
\hline $\mathcal{I}_{X}$ & infinite (Thm 2.5) & infinite (Thm 3.5) & cyclic (Thm 4.3) \\
\hline $\mathcal{S}_{X}$ & infinite & infinite & infinite (Thm 5.1) \\
\hline
\end{tabular}

Table 1: Summary of results.

Diagonal acts were first mentioned, implicitly, in [1]. It was then known that the diagonal right act of $\mathcal{T}_{\mathfrak{N}}$, the full transformation semigroup on the natural numbers, is cyclic. The notion of diagonal acts was then considered by Robertson, Ruškuc and Thomson [5] in relation to wreath products. Necessary and sufficient conditions for the finite generation and presentability of a restricted wreath product were proved, and these referred to the finite generation of diagonal acts. In [4] the same authors considered diagonal acts in their own right and made some interesting connections with finitary power semigroups. It was shown that the diagonal left act of $\mathcal{T}_{\mathbf{N}}$ is cyclic, and that both $R_{N}$ (the monoid of partially recursive functions of one variable) and a particular homomorphic pre-image of $R_{\mathrm{N}}$ have both cyclic diagonal left and right acts. We now search for more semigroups with these properties.

In [2] several statements were proved regarding the classes of infinite semigroups which admit finitely generated diagonal acts. The diagonal bi-act of a non-trivial semigroup is not cyclic if the semigroup is either finite or cancellative. The diagonal bi-act of an infinite semigroup is not finitely generated if it is in any of the following classes: commutative; idempotent; or Bruck-Reilly extensions. Neither the diagonal right nor left act of an infinite semigroup is finitely generated if it is in any of the following classes: left cancellative; right cancellative; inverse; completely regular; completely zero-simple; or locally finite. Clearly there are many families of transformation semigroups that are not in any of these classes, so this is a good place to search for more examples of infinite semigroups with finitely generated diagonal acts.

Throughout the paper we let $X$ be an infinite set. We consider the question of finite generation of the diagonal right, left and bi-acts of the followings semigroups: $\mathcal{B}_{X}$ of binary relations; $\mathcal{P}_{X}$ of partial transformations; $\mathcal{T}_{X}$ of full transformations; $\mathcal{F}_{X}$ of full finite-to-one transformations (that is, no infinite subset is mapped to a single point); $\mathcal{I}_{X}$ of partial injective transformations (also called partial bijections); and $\mathcal{S}_{X}$ of bijections on $X$. Our findings are summarised in Table 1. 


\section{Diagonal Right ACts}

THEOREM 2.1. The diagonal right act of $\mathcal{B}_{X}$, the semigroup of binary relations on an infinite set $X$, is cyclic.

Proof: Let $X_{1}$ and $X_{2}$ be disjoint subsets of $X$ with $X=X_{1} \cup X_{2}$ and $|X|$ $=\left|X_{1}\right|=\left|X_{2}\right|$. We fix bijections $a: X \rightarrow X_{1}$ and $b: X \rightarrow X_{2}$, consider them as binary relations

$$
a=\{(i,(i) a): i \in X\}, \quad b=\{(i,(i) b): i \in X\},
$$

and claim that $\mathcal{B}_{X} \times \mathcal{B}_{X}=\{(a, b)\} \mathcal{B}_{X}$.

Select arbitrary $x, y \in \mathcal{B}_{X}$ and construct $u \in \mathcal{B}_{X}$ as

$$
u=\{((i) a, j):(i, j) \in x\} \cup\{((i) b, j):(i, j) \in y\} .
$$

Then $(x, y)=(a u, b u)$ and hence the diagonal right act of $\mathcal{B}_{X}$ is cyclic.

We note that if $x$ and $y$ are both partial (respectively, full, full finite-to-one) transformations then our construction yields $u$ which is also a partial (respectively, full, full finite-to-one) transformation. Hence we have the following results.

COROLlaRY 2.2. The diagonal right act of $\mathcal{P}_{X}$, the semigroup of partial transformations on an infinite set $X$, is cyclic.

COROLlary 2.3. The diagonal right act of $\mathcal{T}_{X}$, the semigroup of full transformations on an infinite set $X$, is cyclic.

( $T$ his is an extension of the known facts for $\mathcal{T}_{\mathrm{N}}$.)

COROLlary 2.4. The diagonal right act of $\mathcal{F}_{X}$, the semigroup of full finite-toone transformations on an infinite set $X$, is cyclic.

However, it is not possible to apply this reasoning to extend this result to partial (or full) injective mappings. [2, Theorem 7.4] states that no infinite inverse semigroup has a finitely generated diagonal right act. Therefore we have the following result.

THEOREM 2.5. The diagonal right act of $\mathcal{I}_{X}$, the semigroup of partial injective transformations on an infinite set $X$, is not finitely generated.

We also know, from Theorem 5.1 below, that the diagonal right act of $\mathcal{S}_{X}$, the semigroup of bijections on an infinite set $X$, is not finitely generated.

\section{Diagonal Left Acts}

In an analogous manner to the proof of Theorem 2.1 (reversing each pair in each element of $\mathcal{B}_{X}$ ) the next theorem follows. 
TheOREM 3.1. The diagonal left act of $\mathcal{B}_{X}$, the semigroup of binary relations on an infinite set $X$, is cyclic.

This time, however, we cannot use this proof to extend this result to $\mathcal{P}_{X}, \mathcal{T}_{X}$ or $\mathcal{F}_{X}$; reversing a transformation does not yield a transformation. We turn to $\mathcal{T}_{\dot{X}}$, and use a different method of proof. We note that this is another extension of the known facts for $\mathcal{T}_{\text {N }}$.

THEOREM 3.2. The diagonal left act of $\mathcal{T}_{X}$, the semigroup of full transformations on an infinite set $X$, is cyclic.

Proof: We fix a bijection $g: X \rightarrow X \times X$, let $f$ be its inverse and define $p_{1}, p_{2}$ : $X \times X \rightarrow X$ as projections onto the first and second co-ordinates, respectively. We let $a, b \in \mathcal{T}_{X}$ be defined as $a=g p_{1}$ and $b=g p_{2}$, and claim that $\mathcal{T}_{X} \times \mathcal{T}_{X}=\mathcal{T}_{X}\{(a, b)\}$. For $x, y \in \mathcal{T}_{X}$, we define $u \in \mathcal{T}_{X}$ as $(i) u=((i) x,(i) y) f$ for all $i \in X$. Then $(x, y)=(u a, u b)$, so the diagonal left act of $\mathcal{T}_{X}$ is cyclic.

We now turn to $\mathcal{P}_{X}$. As is customary we shall write $(i) x=-$ when $x$ is not defined on $i$; that is, when $i \notin \operatorname{Dom}(x)$. Thus we may consider $\mathcal{P}_{X}$ as the subsemigroup of $\mathcal{T}_{X \cup\{-\}}$ which consists of all the transformations $x$ with $(-) x=-$. If we apply the proof of Theorem 3.2 to $\mathcal{T}_{X \cup\{-\}}$ ensuring that $(-) g=(-,-)$, then it is clear that $a, b \in \mathcal{P}_{X}$, and that if $x, y \in \mathcal{P}_{X}$ then $u \in \mathcal{P}_{X}$. Thus we have the following result.

CoRollary 3.3. The diagonal left act of $\mathcal{P}_{X}$, the semigroup of partial transformations on an infinite set $X$, is cyclic.

By way of contrast, we have the following statement.

THEOREM 3.4. The diagonal left act of $\mathcal{F}_{X}$, the semigroup of full finite-to-one transformations on an infinite set $X$, is not finitely generated.

Proof: Assume that the diagonal left act of $\mathcal{F}_{X}$ is finitely generated, so there is a finite $A \subseteq \mathcal{F}_{X} \times \mathcal{F}_{X}$ such that $\mathcal{F}_{X} \times \mathcal{F}_{X}=\mathcal{F}_{X} A$. For all $x, y \in \mathcal{F}_{X}$ there are $u \in \mathcal{F}_{X},(a, b) \in A$ such that $(x, y)=(u a, u b)$. It is clear that

$$
\left\{((k) x,(k) y): k \in X, x, y \in F_{X}\right\}=X \times X
$$

so we have

$$
\{((k) a,(k) b): k \in X,(a, b) \in A\}=X \times X
$$

Fixing $l \in X$, this implies that

$$
\{l\} \times X \subseteq\{((k) a,(k) b): k \in X,(a, b) \in A\} .
$$

As $A$ is finite, there is a particular $(a, b) \in A$ and infinite $Y \subseteq X$ such that

$$
\{l\} \times Y \subseteq\{((k) a,(k) b): k \in X\}
$$


so $l \in X$ has infinitely many pre-images under $a$, and hence $a \notin \mathcal{F}_{X}$, a contradiction.

Thus $\mathcal{F}_{X}$ is the only one of our semigroups for which the diagonal right and left acts behave differently. Other known examples of semigroups with finitely generated diagonal right but not left acts are defined by using certain semigroup constructions, in particular, 'constant extensions' (discussed in [4]) and direct products with finite left zero semigroups (discussed in [2]).

The dual of [2, Theorem 7.4] states that no infinite inverse semigroup has a finitely generated diagonal left act. Therefore we have the following result.

THEOREM 3.5. The diagonal left act of $\mathcal{I}_{X}$, the semigroup of partial injective transformations on an infinite set $X$, is not finitely generated.

We also know, from Theorem 5.1 below, that the diagonal left act of $\mathcal{S}_{X}$, the semigroup of bijections on an infinite set $X$, is not finitely generated.

\section{Diagonal Bi-Acts}

As the diagonal right acts of $\mathcal{B}_{X}, \mathcal{P}_{X}, \mathcal{T}_{X}$ and $\mathcal{F}_{X}$ ( $X$ infinite) are cyclic, it follows that the diagonal bi-acts of these semigroups are also cyclic. We now consider $\mathcal{I}_{X}$, the semigroup of partial injective transformations on an infinite set $X$, and show that, in contrast to its diagonal right and left acts, its diagonal bi-act is cyclic.

This semigroup is important in inverse semigroup theory; just as all groups are subgroups of $\mathcal{S}_{X}$ for some set $X$, all inverse semigroups are subsemigroups of some $\mathcal{I}_{X}$. In [3], the notion of cycle representations of permutations is extended to that of path representations of partial injective transformations. We shall use this notion, in an infinite context, in the proof of our result.

For $x \in \mathcal{I}_{X}$ we let $\Gamma_{x}$ be a digraph with vertex set $X$ and edges specified by

$$
i \rightarrow j \Leftrightarrow(i) x=j \text {. }
$$

Let $\Omega_{X}$ be the set of all digraphs on $X$ in which every vertex has in-degree either 0 or 1 and out-degree either 0 or 1 . Then there is a natural bijection $\phi: \mathcal{I}_{X} \rightarrow \Omega_{X}$, defined as $(x) \phi=\Gamma_{x}$.

For graphs $\Lambda_{1}$ and $\Lambda_{2}$, a graph isomorphism $\psi: \Lambda_{1} \rightarrow \Lambda_{2}$ is a bijection between the vertex sets such that

$$
i \rightarrow j \text { in } \Lambda_{1} \Leftrightarrow(i) \psi \rightarrow(j) \psi \text { in } \Lambda_{2} .
$$

If there exists a graph isomorphism from $\Lambda_{1}$ to $\Lambda_{2}$ then they are isomorphic, which is denoted $\Lambda_{1} \cong \Lambda_{2}$.

We say that $p, q \in \mathcal{I}_{X}$ are conjugate if there exists $s \in \mathcal{S}_{X}$ (the group of bijections on $X$ ) such that $q=s p s^{-1}$. In [3] it is shown, for a finite set $X$, that $p, q \in \mathcal{I}_{X}$ are conjugate if and only if they have the same path structure. We state and prove this in terms of digraphs, and where $X$ may be infinite or finite. 
LEMMA 4.1. Elements $p, q \in \mathcal{I}_{X}$ are conjugate if and only if $\Gamma_{p}$ and $\Gamma_{q}$ are isomorphic.

Proof: $(\Rightarrow)$ If there is $s \in \mathcal{S}_{X}$ such that $q=s p s^{-1}$, then

$$
\begin{aligned}
i \rightarrow j \text { in } \Gamma_{p} \Leftrightarrow(i) p=j \Leftrightarrow\left[(i) s^{-1}\right] s p s^{-1}=(j) s^{-1} & \\
& \Leftrightarrow\left[(i) s^{-1}\right] q=(j) s^{-1} \Leftrightarrow(i) s^{-1} \rightarrow(j) s^{-1} \text { in } \Gamma_{q}
\end{aligned}
$$

so $s^{-1}: \Gamma_{p} \rightarrow \Gamma_{q}$ is a graph isomorphism.

$(\Leftarrow)$ A graph isomorphism $\psi: \Gamma_{p} \rightarrow \Gamma_{q}$ is a bijection on $X$, so may be considered as some $s \in \mathcal{S}_{X}$. Also,

$$
\begin{aligned}
(i) p=j \Leftrightarrow i \rightarrow j \text { in } \Gamma_{p} \Leftrightarrow(i) \psi \rightarrow(j) \psi \text { in } \Gamma_{q} & \\
& \Leftrightarrow[(i) \psi] q=(j) \psi \Leftrightarrow(i) s q=(j) s \Leftrightarrow(i) s q s^{-1}=j
\end{aligned}
$$

so $p=s q s^{-1}$ and they are conjugate.

The components of a digraph are the connected components of its underlying undirected graph. Up to isomorphism, the only components which may appear in $\Lambda \in \Omega_{X}$ are:

1. finite paths of size $r$ (for all $r \in \mathbb{N}$ ) $i_{1} \rightarrow i_{2} \rightarrow \cdots \rightarrow i_{r}$;

2. finite cycles of size $r$ (for all $r \in \mathbb{N}$ ) $i_{1} \rightarrow i_{2} \rightarrow \cdots \rightarrow i_{r} \rightarrow i_{1}$;

3. left infinite paths $\cdots \rightarrow i_{-2} \rightarrow i_{-1} \rightarrow i_{0}$;

4. right infinite paths $i_{1} \rightarrow i_{2} \rightarrow i_{3} \rightarrow \cdots$;

5. bi-infinite paths $\cdots \rightarrow i_{-1} \rightarrow i_{0} \rightarrow i_{1} \rightarrow \cdots$.

In particular, there are only countably many isomorphism classes for components, so these can be indexed by $\mathbb{N}$. To be more precise, we fix a family $\left\{R_{n}: n \in \mathbb{N}\right\}$ where $R_{n}$ is a component in some digraph $\Lambda_{n} \in \Omega_{X}$ and such that for any component $C$ in any $\Lambda \in \Omega_{X}$ there is a unique $n \in \mathbb{N}$ such that $C \cong R_{n}$. In this case we refer to $n$ as the isomorphism class of $C$.

The restriction of $p \in \mathcal{I}_{X}$ to $Y \subseteq X$ is denoted $p \uparrow_{Y}$ and defined as $(i) p \uparrow_{Y}=(i) p$ if $i,(i) p \in Y$ and $\left.(i) p\right|_{Y}=-$ otherwise. It is clear that $\left.p\right|_{Y} \in I_{X}$.

LEMMA 4.2. There exists $c \in \mathcal{I}_{X}$ such that for all $p \in \mathcal{I}_{X}$ there exists $s \in \mathcal{I}_{X}$ such that $p=s c s^{-1}$.

Proof: We let $Z$ be an index set with $|Z|=|X|$ and partition $X$ as the disjoint union

$$
X=\bigcup_{z \in Z, n \in N} V_{z, n}
$$

where each $\left|V_{z, n}\right|=\left|R_{n}\right|$. We let $\Upsilon \in \Omega_{X}$ be arbitrary such that each $V_{z, n}$ is the vertex set of a component of isomorphism class $n$ and let $c \in \mathcal{I}_{X}$ be such that $\Gamma_{c}=\Upsilon$. 
Let $p \in \mathcal{I}_{X}$ be arbitrary and let $F_{q}(q \in Q)$ be the components of $\Gamma_{p}$. As every graph in $\Omega_{X}$ has at most $|X|$ components of each isomorphism class, we may assume without loss of generality that $Q \subseteq Z$. For each $F_{q}$ we consider $V_{q, n}$, where $n$ is the isomorphism class of $F_{q}$. Clearly $F_{q} \cong V_{q, n}$, so the restrictions $\left.p\right|_{F_{q}}$ and $\left.c\right|_{V_{q, n}}$ are conjugate, by Lemma 4.1. That is, there exists $s_{q} \in \mathcal{S}_{X}$ such that $\left.p\right|_{F_{q}}=\left.s_{q} c\right|_{V_{q, n}} s_{q}^{-1}$. We define $s: X \rightarrow X$ as $(i) s=(i) s_{q}$ when $i \in F_{q}$. Then $s$ is a well-defined full injective (but not necessarily surjective) transformation and $p=s c s^{-1}$.

We are now ready to prove the main result.

THEOREM 4.3. The diagonal bi-act of $\mathcal{I}_{X}$, the semigroup of partial injective transformations on an infinite set $X$, is cyclic.

Proof: We claim that $\mathcal{I}_{X} \times \mathcal{I}_{X}=\mathcal{I}_{X}\{(c, 1)\} \mathcal{I}_{X}$ where $c$ is as in Lemma 4.2. Let $x, y \in \mathcal{I}_{X}$ be arbitrary. By Lemma 4.2 there exists $s \in \mathcal{I}_{X}$ such that $x y^{-1}=s c s^{-1}$, and we note that

$$
\text { (i) } x=(j) y \Leftrightarrow(i) x y^{-1}=j \Leftrightarrow(i) s c s^{-1}=j \Leftrightarrow(i) s c=(j) s .
$$

Let $t: X \rightarrow X$ be defined on $\operatorname{Im}(s c) \cup \operatorname{Im}(s)$ such that $[(i) s c] t=(i) x$ and $[(j) s] t=(j) y$.

Suppose that a contradiction is implicit here, in that $t$ is required to map one point to two different image points. This could only be the case if $(i) s c=(j) s$ but $(i) x \neq(j) y$ for some $i, j \in X$, which by (1) cannot happen.

Suppose that $t$ is not injective. This is only the case if $(i) x=(j) y$ but $(i) s c \neq(j) s$ for some $i, j \in X$. Again, by (1), this does not happen.

So $t \in \mathcal{I}_{X}$ and $(x, y)=(s c t, s t)=s(c, 1) t$, and the theorem is proved.

\section{Further Remarks and Conclusion}

In the course of this paper we considered many families of transformation semigroups. For $\mathcal{S}_{X}$, the symmetric group on an infinite set $X$, we use the following result, which appears as [6, Proposition 6.7] and which we reprove here for completeness.

THEOREM 5.1. If $G$ is a group then the diagonal bi-act of $G$ is finitely generated if and only if $G$ has only finitely many conjugacy classes.

Proof: $(\Rightarrow)$ If the diagonal bi-act of $G$ is finitely generated then there is a finite $U \subseteq G$ such that $G \times G=G(U \times U) G$. For all $x \in G$ there are $u_{1}, u_{2} \in U, g_{1}, g_{2} \in G$ such that $\left(x, 1_{G}\right)=g_{1}\left(u_{1}, u_{2}\right) g_{2}$. Then $x=1_{G}^{-1} x=g_{2}^{-1} u_{2}^{-1} g_{1}^{-1} g_{1} u_{1} g_{2}=g_{2}^{-1}\left(u_{2}^{-1} u_{1}\right) g_{2}$ is conjugate to $u_{2}^{-1} u_{1}$, so $G$ has only finitely many conjugacy classes.

$(\nLeftarrow)$ If $G$ has only finitely many conjugacy classes, then let $U$ be a set of conjugacy class representatives in $G$. For arbitrary $x, y \in G$ there are $u \in U, g \in G$ such that $x y^{-1}=g^{-1} u g$, so

$$
(x, y)=g^{-1}\left(u, 1_{G}\right) g y \in G(U \times U) G,
$$


and we see that $U \times U$ finitely generates the diagonal bi-act of $G$.

It is now clear that the diagonal bi-act of $\mathcal{S}_{X}$ is not finitely generated. Without proof, we also remark that none of the following semigroups: injections; non-injections; surjections; non-surjections; or partial surjections on an infinite set have finitely generated diagonal bi-acts.

There are many open problems concerning which infinite semigroups have finitely generated diagonal acts. We know that the semigroup $S$ of all $n \times n$ matrices over an infinite field $F$ has no finitely generated diagonal acts, as $F$ (under multiplication) is a homomorphic image of $S$ and would inherit these properties; it is clear from [2] that this is not the case. We ask if the semigroup $S$ of all $n \times n$ matrices over an infinite ring $R$ can have any finitely generated diagonal acts? Again, $R$ (under multiplication) is a homomorphic image of $S$ so we ask: does there exist an infinite ring $R$ whose multiplicative semigroup $R^{*}$ has a finitely generated diagonal right, left or bi-act?

\section{REFERENCES}

[1] S. Bulman-Fleming and K. McDowell, 'Problem e3311', Amer. Math. Monthly 96 (1989).

[2] P. Gallagher, 'On the finite and non-finite generation of diagonal acts', Comm. Algebra (to appear).

[3] S. Lipscomb, Symmetric inverse semigroups (American Mathematical Society, Providence R.I., 1996).

[4] E.F. Robertson, N. Ruskuc and M.R. Thomson, 'On diagonal acts of monoids', Bull. Austral. Math. Soc. 63 (2001), 167-175.

[5] E.F. Robertson, N. Ruskuc and M.R. Thomson, 'On finite generation and other finiteness conditions of wreath products of semigroups', Comm. Algebra 30 (2002), 3851-3873.

[6] M.R. Thomson, Finiteness conditions of wreath products of semigroups and related properties of diagonal acts, (Ph.D. Thesis) (University of St Andrews, St. Andrews, Scotland, 2001).

School of Mathematics and Statistics

University of St. Andrews

North Haugh

St. Andrews

Scotland KY16 9SS 